GENOMICS

\section{A TRIBE for RNA}

Cell 165, 742-753 (2016)

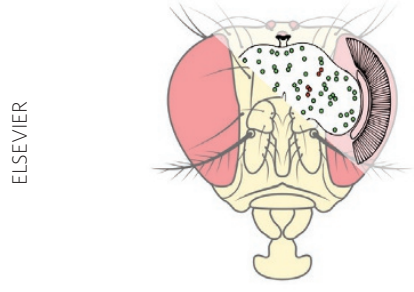

Although the cross-linking and

immunoprecipitation approach (CLIP) has been used to find targets of RNA-binding proteins (RBPs) in cells, the application of this method to identify cell-type-specific RBP targets in vivo remains limited because of the large amount of cellular material required. McMahon et al. developed a method called TRIBE (targets of RNAbinding proteins identified by editing) that allows cell-specific identification of RBPRNA complexes in Drosophila using small amounts of RNA. They coupled an RBP to the catalytic domain of the RNA-editing enzyme ADAR that converts adenosine to inosine, which is subsequently read as guanosine. These editing events can be identified through RNA sequencing and bioinformatics analysis. As an example, the authors used TRIBE to identify targets of an RBP, Hrp48, and confirmed the detection of the same known RNA targets as for CLIP analysis in a Drosophila cell culture line. Hrp48-TRIBE was then expressed in Drosophila neuronal cell types ranging from dopaminergic neurons (containing 1,000 cells) to the small circadian PDF-neuropeptide-expressing cells
(16 cells). In addition to detecting Hrp48 targets found in all neuronal cell types, McMahon et al. also identified unique cell-type-specific Hrp48 targets, which will prompt further analysis. Taken together, these results validate TRIBE as a useful tool that will help unravel the riboproteome of individual cell types. $G M$

PROTEIN EVOLUTION

\section{Polymerase's new language \\ Nat. Chem. http://dx.doi.org/10.1038/ nchem.2493 (2016)}

DNA aptamers are of potential use in numerous biotechnological applications, and the addition of methoxy (OMe) or fluorine substituents to $\mathrm{C}^{\prime}$ ' of the sugar ring makes DNA resistant to nucleasemediated degradation. However, natural DNA polymerases can neither read sequences containing these substituents nor incorporate the modified nucleotides into newly synthesized oligomers, which hampers their use in aptamer evolution. Using an improved phage-based selection system for evolved polymerases, Chen et al. identified novel thermostable DNA polymerase variants that can 'reverse transcribe' a fully C2'-OMe-modified template into a DNA product or vice versa. A third polymerase variant identified in the screen can directly PCR amplify DNA with every G, C, A, or U nucleotide modified with $\mathrm{C} 2$ '-OMe or any combination of two nucleotides modified with $\mathrm{C} 2$ '-F. Analysis of the mutations acquired during polymerase evolution indicates that their increased activity with unnatural substrates stems from stabilization of the interaction between the finger and thumb domains via a salt bridge. The error rates for these evolved enzymes are 10- to

\section{HOST-MICROBE INTERACTIONS}

\section{Inflammation sop-up PLoS Pathog. http://dx.doi.org/10.1371/journal.ppat.1005552 (2016)}

Salmonella typhimurium utilizes a type III secretion system to deliver bacterial effector proteins such as the E3 ligase SopA into host cells. These effectors induce inflammation in the host and co-opt host function in the intestinal epithelium. In order to understand how SopA effects changes in host signal transduction pathways that lead to inflammation, Kamanova et al. first used affinity purification to identify two host E3 ubiquitin ligases, TRIM56 and TRIM65, as SopA-binding proteins during infection. SopA enzymatic activity enhanced TRIM65 ubiquitylation but did not target TRIM65 for proteasome-mediated degradation. Instead, the authors found that TRIM 65 ubiquitin ligase activity was required to promote interactions with the innate immune receptor MDA5 to modulate expression of the proinflammatory cytokine interferon- $\beta$. SopA also increased the ability of TRIM56 to stimulate interferon- $\beta$ expression through RIG-1, another innate immune receptor, and increased the expression of other proinflammatory cytokines in mice infected with S. typhimurium. These results suggest a mechanism whereby bacterial SopA acts through host ubiquitin ligases to mediate host inflammation via two major innate immune receptor pathways, and benefiting the bacteria by providing essential nutrients for growth.

Cis-acting RNA elements in bacterial genes, such as riboswitches and attenuators, regulate gene expression in response to metabolite binding. To date, most riboregulators and their ligands have been discovered by comparative genomic analysis calibrated with metabolic logic. Dar et al. now report an approach, called term-seq, for the experimental identification of riboregulators and their modes of gene regulation. Term-seq maps the 3 '-termini of RNA transcripts on a genome-wide scale and, when used in combination with other genomic methods, identifies genes that contain putative riboregulator motifs and undergo conditional transcriptional termination in response to the presence of a metabolite or drug. In Bacillus subtilis and other bacteria, the method successfully identified known riboregulators and revealed previously unknown smallmolecule-dependent regulatory RNA motifs. For example, in the pathogenic bacterium Listeria monocytogenes, a small RNA, rli53, was identified as a regulator of the bacterial response to the antibiotic lincomycin: in the presence of drug, this riboregulator facilitated transcription of lmo0919, an ABC transporter that acts as an antibiotic efflux pump. A meta-transcriptomic analysis of human microbiome samples demonstrated the utility of term-seq for profiling antibioticresponsive riboregulator activity within microbial communities, greatly expanding the scope of this tool for RNA motif discovery and antimicrobial research. 